LM-00K023

May 2, 2000

\title{
Thermal Diffusivity and Conductivity in Ceramic Matrix Fiber Composite Materials - Literature Study
}

\author{
R.G. Quinn
}

\section{NOTICE}

This report was prepared as an account of work sponsored by the United States Government. Neither the United States, nor the United States Department of Energy, nor any of their employees, nor any of their contractors, subcontractors, or their employees, makes any warranty, express or implied, or assumes any legal liability or responsibility for the accuracy, completeness or usefulness of any information, apparatus, product or process disclosed, or represents that its use would not infringe privately owned rights. 


\title{
Thermal Diffusivity and Conductivity in Ceramic Matrix Fiber Composite Materials - Literature Study
}

\author{
By
}

Richard G. Quinn

A technical literature review was conducted to gain an understanding of the state of the art method, problems, results, and future of thermal diffusivity/conductivity of matrix-fiber composites for high temperature applications. This paper summarizes the results of test method development and theory. Results from testing on various sample types are discussed with concentration on the anisotropic characteristics of matrix-fiber composites, barriers to heat flow, and notable microstructure observations. The conclusion presents some observations from the technical literature, drawbacks of current information and discusses potential needs for future testing.

\section{Introduction}

Ceramic matrix-fiber composite materials have distinct advantages over conventional materials for high temperature applications. Testing is in progress to better understand and predict the behavior of ceramic materials in high temperature environs. Poor ductile behavior of ceramic materials has led to the development of fiber-reinforced ceramic materials in an attempt to toughen these materials. Mechanical properties of ceramic matrix-fiber composites have been and are still being extensively studied. Emphasis on thermal properties studies of these materials is becoming more prevalent. The introduction of fibers results in anisotropic behavior in both the mechanical and thermal properties.

The understanding of two thermal properties (diffusivity and conductivity) is important in order to take full advantage of these materials in high temperature applications. Analytical studies have shown that the behavior of these two properties is a 
function of the material type, volume percentages of the constituents, phase distribution, direction of heat flow, types of fibers, angles of fibers and basic properties of the constituent materials. Theories developed to describe the thermal behavior of ceramic matrix-fiber composites cannot rely solely on the rule of mixtures. Fabrication methods, coating applications, impurities, degree of dissimilar component contact, interfacial thermal barriers, and defects have all contributed to the difficulties in predicting the thermal behavior of these composites. In almost all cases, these factors lead to a lowering of the thermal conductivity of the composite.

\section{Experimental Methods}

A variation of the longitudinal transient heat flow method is the primary method that is used to obtain diffusivity data. Commonly referred to as the flash method, this method was developed by Parker, Jenkins, Butler and Abbott in $1961^{\prime}$. The flash method has advantages over other methods and is a good choice for sample analysis performed at high temperature. Parker et al found that the flash method eliminated the problem of thermal contact resistance, and heat losses were minimized because the measurement time period was so short that very little cooling took place. Additionally, the back surface temperature did not begin to change during the heating pulse. Thus, the sample can be treated as an infinite slab despite being very thin. For diffusivity measurements, the amount of energy absorbed on the front surface does not have to be known. This value of the energy absorbed is only needed to determine specific heat or conductivity values via the flash method. The following equation is used to calculate diffusivity by the flash method, where $\alpha$ is diffusivity, $\ell$ is thickness, and $t_{1 / 2}$ is the time when the back of 
the sample reaches half its maximum temperature.

$$
\alpha=1.38 \ell^{2} / \pi^{2} t 1 / 2
$$

Samples are often coated to darken their surface. This prevents irradiation of the back of the sample, increases the amount of energy absorbed during the flash pulse, and ensures that the absorptivity of all samples is identical. All references listed in this paper used the flash method for composite diffusivity or conductivity measurements.

Diffusivity is related to conductivity by the following equation,

$$
K=-\alpha \rho C_{p}
$$

where $\mathbf{K}$ is conductivity, $\alpha$ is diffusivity, $\rho$ is density, and $C_{p}$ is specific heat.

Parker et al took heat losses via radiation and convection into consideration when they used the flash method. R.C. Heckman ${ }^{2}$ in 1972 published a paper which further discussed radiant and convective heat losses from the sample face. He went on to consider a coupling of heat loss and finite pulse width effects on the measurement results. Heckman's analysis used the basic theories and he provided corrections in tabular form for the aforementioned effects.

Hasselman and Donaldson ${ }^{3}$ also investigated specimen size and detector nonlinearity effects on the flash method. In earlier studies, they found that different specimen thicknesses yielded different diffusivity values for the same material. They went on to study this effect by comparing their results to the diffusivity results obtained by the National Institute of Standards and Technology (NIST) on 8425 graphite. Hasselman and Donaldson's data showed a dependence of diffusivity values on both flash intensity and specimen thickness with values varying by almost a factor of three. The diffusivities were also always lower than the NIST results. When they attenuated the 
flash beam, they were able to show less dependence on specimen thickness and closer agreement with the NIST results. Also by attenuating the pulse the back surface temperature was kept to a smaller range and thus minimized detector non-linearity.

\section{Theory}

Ideally engineers and scientists would like to use a simple formula to predict the effective conductivity or diffusivity of a fiber composite material and forgo expensive testing. This would require single or multiple formulas to address the various possible fiber orientations and directions of heat flow. Orientation and direction of heat flow are important because of the anisotropy of fiber composites. The anisotropy occurs because the conductivity and diffusivity of the composites are lower for heat flow perpendicular to the fibers than parallel to the fibers.

One method used to predict effective conductivity is based on the rule of mixtures for composites and results in the following equations; ${ }^{4}$

$$
\begin{gathered}
K_{c}=K_{m} V_{m}+K_{p} V_{p} \\
{\left[\left(K_{m}-K_{c}\right) /\left(K_{m}+K_{c}\right)\right] V_{m}=\left[\left(K_{c}-K_{p}\right) /\left(K_{c}+K_{p}\right)\right] V_{p}}
\end{gathered}
$$

where $\mathbf{K}$ is conductivity, $\mathbf{V}$ is volume fraction and the subscripts $\mathbf{m}, \mathbf{p}$, and $\mathbf{c}$ apply to the matrix, fiber and composite, respectively. Equation (3) applies when the heat flow is parallel to uniaxially aligned fibers. Equation (4) applies when the heat flow is perpendicular to the uniaxially aligned fibers. Additional derivations of equations 3 and 4 exist for various fiber orientations, weaves, and heat flow direction.

Another method for effective conductivity determination utilizes the unit cell approach. This approach can be used because of the periodic structure of composite materials and lines of symmetry can be located. Unit cells ${ }^{5}$, which are pictured in 
Figures 1 and 2, are indicated by the grayed boxes for a rectangular and staggered pattern of fibers, respectively. The angle that the diagonal of the unit cell makes with the base is

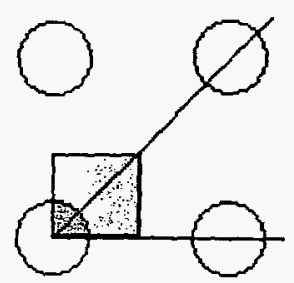

Figure 1 - Unit cell for rectangular pattern of fibers
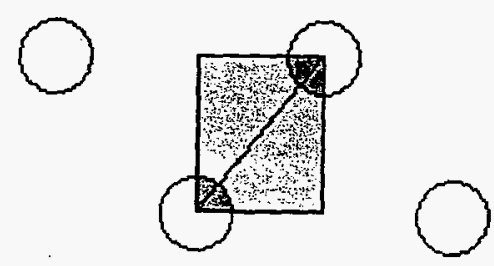

Figure 2 - Unit cell for staggered pattern of fibers

an important part of the solution to the effective conductivity ${ }^{5}$. The unit cell approach solves the following equation; where $\mathbf{K}$ is conductivity and $\mathbf{T}$ is temperature. Equation 5

$$
K_{x x} \frac{\delta^{2} T}{\delta x^{2}}+2 K_{x y} \frac{\delta^{2} T}{\delta x \delta y}+K_{y y} \frac{\delta^{2} T}{\delta y^{2}}=0
$$

and is based on two dimensional heat flow or an infinite $\mathrm{z}$ direction. The solution by the unit cell approach can easily be adapted to tables or graphs of $\mathrm{K}_{\mathrm{eff}} / \mathrm{K}_{\text {matrix }}$ versus $\mathrm{K}_{\text {fiber }} / \mathrm{K}_{\text {matrix }}{ }^{5,6}$ for use in handbooks.

These approaches assume that the conductivities of the individual composite materials are consistent with their values as measured in the pure state. Typical ceramics have conductivities that vary with temperature. They follow an equation of the following form ${ }^{7}$ when temperature is greater than $100^{\circ} \mathrm{C}$, where $\mathrm{K}$ is conductivity, $\mathrm{C}$ is a constant

$$
\mathbf{K}^{-1}=\mathbf{C}+\mathbf{a T}
$$

less than one, $\mathrm{a}$ is a constant in the range of $10^{-6}$ to $10^{-5}$, and $\mathrm{T}$ is temperature in degrees Kelvin. Figure 3 represents the typical shape of the plot from equation (6) for temperatures greater than $100^{\circ} \mathrm{C}$. Conductivity plots based on the theories above show that the effective conductivity for the composite would fall somewhere between the plots of the individual constituents (fiber and matrix). 


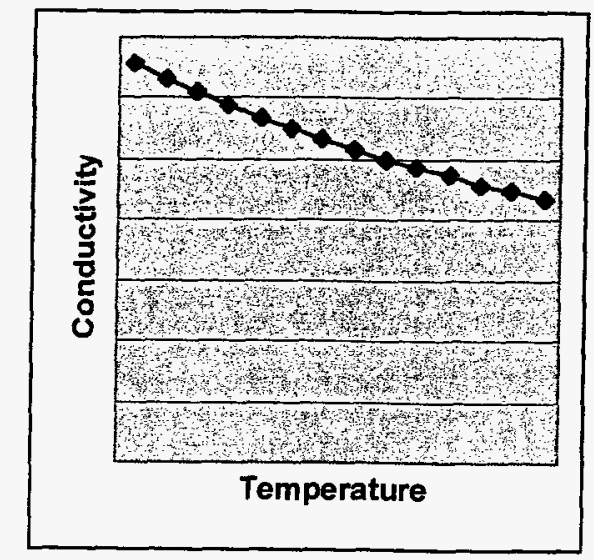

Figure 3 - Typical conductivity-temperature plot for ceramics greater than $100^{\circ} \mathrm{C}$

The approaches discussed above do not always accurately predict composite material conductivity. Several experiments have shown that the conductivity of the fibers can differ drastically from their pure state ${ }^{7}$. The full cause of this is not known but many fibers have been found to have a high number of defects. Both methods work well with metal composites because the heat transport by the electron mechanism is much more efficient than the phonon mechanism in ceramic fiber composites. Predicting the effect of the behaviors discussed in the introduction is difficult without a close inspection of the microstructure to determine the phase distribution, defects, and degree of contact. These effects can then be incorporated into the fundamental equations as additional conductivities or corrections.

\section{Experimental Results}

General Discussion

Many experiments have confirmed the trends predicted by theory despite the fact that individual results do not always agree. exactly. The composite diffusivities and conductivities versus temperature fall between plots of their respective pure constituents, 
as shown for diffusivities in Figure 4. The diffusivities and conductivities of the composite approaches the pure fiber values as the fiber content increases ${ }^{7}$. The

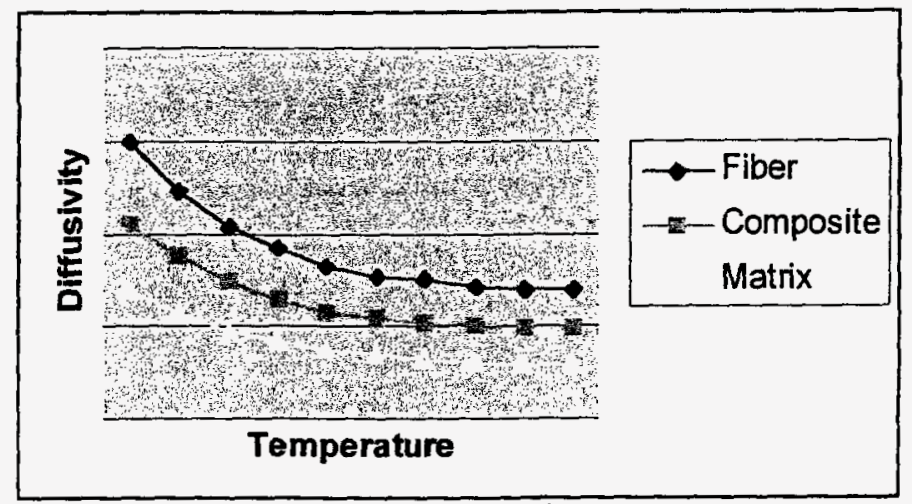

Figure 4 - Typical diffusivity-temperature plot showing progression from matrix to composite to fiber

direction of increasing fiber volume percent is indicated by the progression from light to dark blue of the curves in Figure 4.

\section{Interfacial Barriers}

Interfacial barriers lower conductivity and diffusivity in composites and in the worst case conditions result in thermal values equal to the matrix phase. Likewise, the conductivity and diffusivity values are lower than theoretical predictions. These observations are more pronounced when heat flow is perpendicular to the fiber direction ${ }^{8}$.

The thermal barriers can be due to oxidation layers on fibers ${ }^{8}$, delamination ${ }^{9}$, fiber coatings $^{8}$, and differences in the coefficient thermal expansion (CTE) between the fiber and the matrix ${ }^{10}$. Data taken from reference 8 and presented in Figure 5, show how the diffusivity values for the composite, with interfacial barriers and heat flow perpendicular to the Silicon Carbide fibers, approach the diffusivity of the reaction bonded silicon nitride (RBSN) matrix (closeness of the yellow and blue curves). 


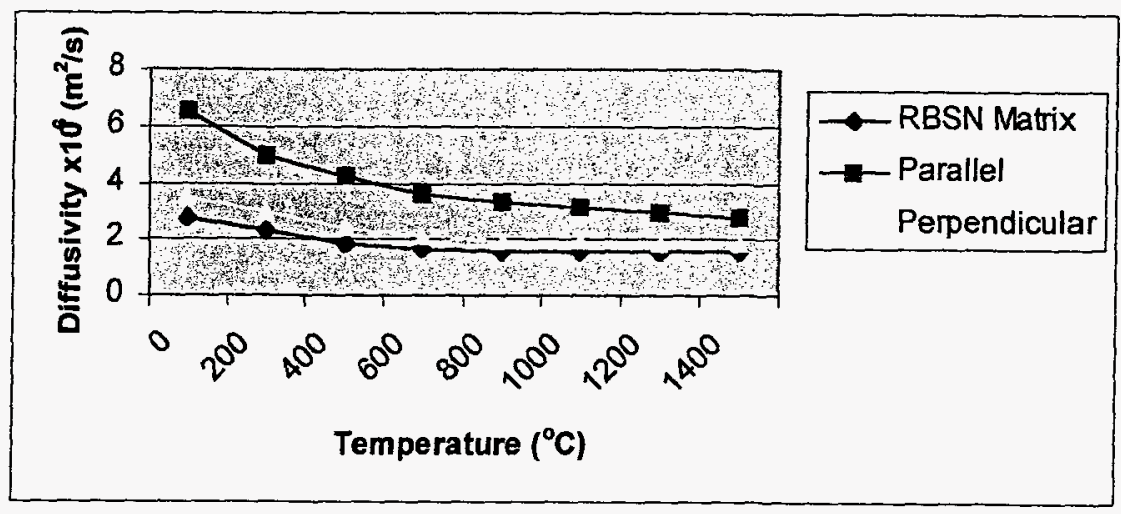

Figu re 5 - Diffusivity-temperature plot for SiC-RBSN composite ${ }^{8}$

Delamination or debonding can be caused by poor processing or in-service failures. This condition presents problems when attempting to predict the thermal behavior of the composite. The gaps and voids are not uniform in any way, which makes it difficult to obtain the detailed geometry information that is essential for modeling. When interfacial barriers are included in theoretical calculations they are included as a conductance term ${ }^{8,10}\left(\boldsymbol{h}_{\mathfrak{i}}\right)$. See equation (7) below which has been derived from equation (4). Conductance is strongly influenced by barrier geometry.

$$
\begin{aligned}
K_{c}= & K_{m}\left[\left(K_{\mathrm{f}} / K_{m}-1-K_{f} / a h_{i}\right) V_{p}+1+K_{f} / K_{m}+K_{f} / a h_{i}\right] / \\
& {\left[\left(1-K_{f} / K_{m}+K / a h_{i}\right) V_{p}+1+K_{f} / K_{m}+K_{f} / a h_{i}\right] }
\end{aligned}
$$

CTE mismatch may result in small gaps between the fiber and the matrix or can lead to fiber coating failures. These gaps can be difficult to see even with a scanning electron microscope. Calculations using the CTEs of the respective composite materials can predict gaps as small as $0.1 \mu \mathrm{m}^{11}$. Diffusivity measurements conducted in different gaseous environments and in a vacuum for the same material have suggested that gaps existed and that the heat flow was affected by the different values of heat conduction of the respective gas ${ }^{11}$. The existence of small gaps was also suggested by conducting diffusivity measurements at constant temperature. A plot similar to Figure 6 
was observed. The flat red portion of the curve represents the portion of the test performed at vacuum. The atmosphere was changed to nitrogen at the start of the blue curve and starts to flatten out within ten minutes. This curve has the shape of the charging rate of an electrical capacitor. In tests of this same sample in different atmospheres, the vacuum atmosphere plot showed a sharp diffusivity increase as the nitriding temperature was approached, suggesting a closure of the gap between the fiber and the matrix ${ }^{11}$.

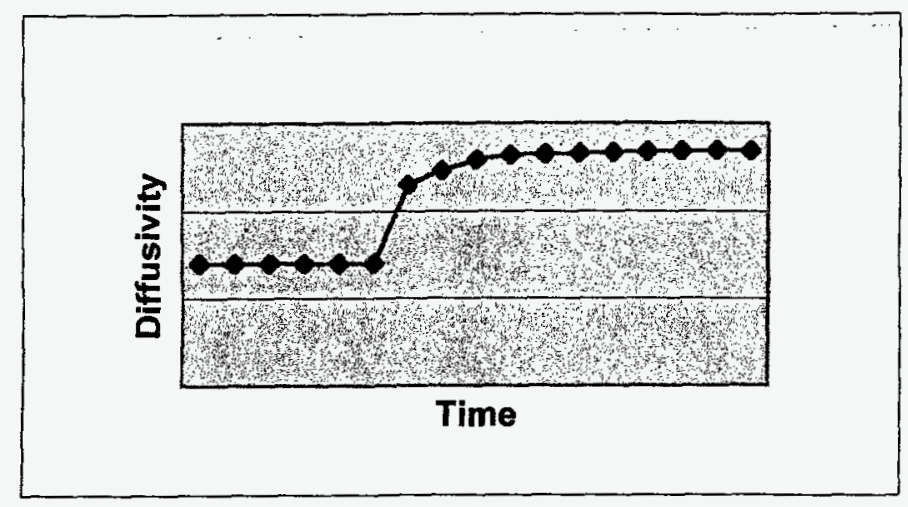

Figure 6 - Diffusivity-time plot at constant temperature in changing atmosphere

\section{Microstructure Effects}

The anisotropic behavior of matrix-fiber composites has already been discussed. This behavior can be observed in mechanical properties and was illustrated in thermal properties in Figure 5. The word composite implies that there will be some different phases involved, matrix and fibers. The designer may wish to take advantage of the orientation of these two phases and their individual properties. A fiber with high conductivity characteristics could be oriented perpendicular to the heat flow in a low conductivity matrix ${ }^{12}$. In this case the heat energy can be diverted along the fibers and carried to other components or heat exchangers and possibly be recovered. 
Phase differences have been observed in various matrix and fiber materials. One particular phase condition that has been noted is preferred orientation. Some other examples are as follows:

1. Whiskers have been shown to preferentially orient in a direction perpendicular to the hot pressing direction ${ }^{7,13}$. This condition was not observed in isostatically pressed samples.

2. In silicon nitride the elongated $\beta$-phase was noted to be preferentially oriented in a direction perpendicular to the hot pressing direction. This condition coincided with the crystal's $c$ axis ${ }^{14}$.

3. The boron nitride (BN) (matrix phase) in silicon carbide-boron nitride composites have been shown to be preferentially oriented with the $\mathrm{c}$ axis parallel to the direction of the hot pressing direction while the $\beta$-phase of the silicon carbide was found to be preferentially oriented in a direction perpendicular to the pressing direction ${ }^{15}$.

Within the fibers of silicon nitride $\left(\mathrm{Si}_{3} \mathrm{~N}_{4}\right)$, the ratio of $\alpha$-phase to $\beta$-phase is important. The $\alpha$-phase has small erratic grains with short mean free phonon paths relative to $\beta$ phase, resulting in lower conductivity values ${ }^{13}$. The $\beta$-phase has larger equiaxed grains. Higher temperatures and pressures during processing result in more and larger $\beta$-phase grains oriented perpendicular to the pressing direction ${ }^{13,16}$. This aging condition has been noted in hot pressed powdered metals. Diffusivity measurements made during a heating and cooling cycle showed a hysteresis effect in a SiC-SiC composite ${ }^{16}$, Figure 7. The up to three times increase in diffusivity was due to grain growth of $\beta$-phase SiC. Another 


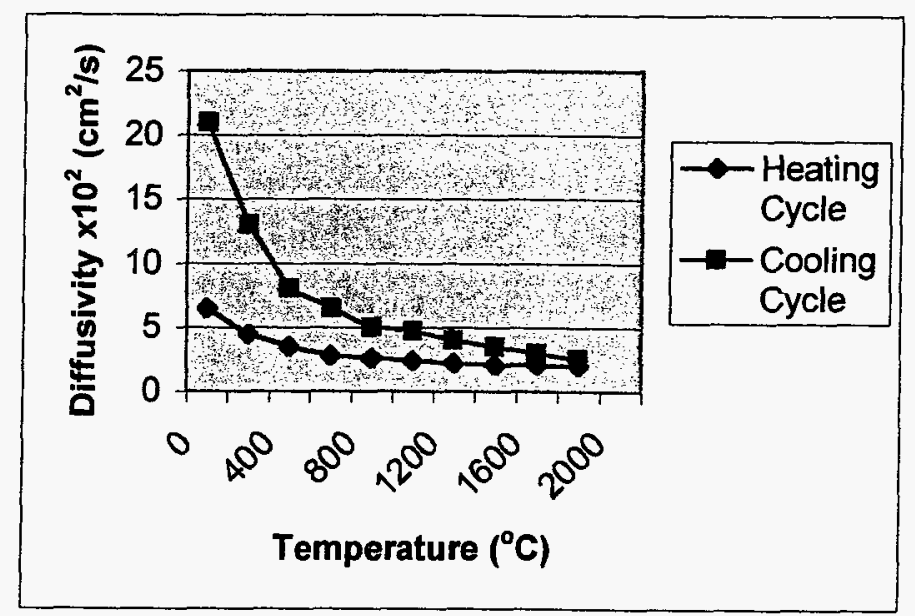

Figure 7 - Diffusivity-temperature plot for a SiC-SiC composite. Cooling cycle followed a $3 \mathrm{hr}$ hold at $1800^{\circ} \mathrm{C}$

condition believed to be occurring to cause this change was impurities reacting to form carbon monoxide. A mass loss was noted to occur during the experiment.

Processing and fabrication of ceramic matrix composites is as important to microstructure as it is in metals. In reaction sintered bonded $\mathrm{Si}_{3} \mathrm{~N}_{4}$, an abundance of strained $\alpha$-phase exists and an overall lower diffusivity and conductivity in the parallel and perpendicular directions was observed than in the hot pressed $\mathrm{Si}_{3} \mathrm{~N}_{4}$. The drastic difference in $\mathrm{SiC}$ fiber conductivities is an excellent example of processing effects. Several processes exist for manufacturing $\mathrm{SiC}$ fibers and all the resultant fibers have different conductivity values. The ability to measure conductivity or diffusivity of independent fibers or whiskers does not currently exist. Some of the values listed below in Table I were determined by back calculating from known matrix and respective composite conductivities. Many of the references credit defects or the lack of defects for the differences observed in Table I. Stacking faults appear to be the major contributor ${ }^{17,18}$ while impurities also play a big part in the differences ${ }^{17}$. 
Other general microstructure effects that have been noted in matrix-fiber composites include:

1. Higher density composites have respectively higher diffusivities in both the parallel and perpendicular directions.

2. High fault densities in fibers equate to lower diffusivity and conductivity and is more pronounced in the direction of the fiber than perpendicular to them ${ }^{18}$.

TABLE I - SiC diffusivity values from different references

SiC Type Diffusivity $\left(\mathrm{W} / \mathrm{m}^{\circ} \mathrm{K}\right)$

Vapor-Liquid-Solid (VSL) $^{17} \quad 100-250$

Rice Hull ${ }^{7} \quad 60$

$\mathrm{SiC}^{9 *} \quad 50$

Rice Hull $^{18} \quad 26$

Vapor-Solid (VS) $^{17} \quad 20$

Chemical Vapor Deposition (CVD) ${ }^{15} \quad 1.5$

* no particular process or source was identified in the reference

\section{Discussion}

The diffusivity/conductivity of ceramic matrix composites can be determined through experimental methods. The test result trends have shown close agreement with trends predicted by theory. Some tests of composite materials showed very close agreement with theory, while others deviated appreciably due to interfacial barriers or microstructure effects. The varied non-compiled data presented in Table I indicates that 
there is a need to characterize matrix-fiber composites in terms of parameters such as, composition, density, method of fabrication, thermal history, mechanical and thermal properties. Since there are so many possible combinations, the designer will have to start with preliminary matrix-fiber mixes and determine if the material has already been characterized. The designers may have to support a carefully controlled test program to obtain the desired thermal performance data. The focus of the test program should be to understand the effects of microstructure and possible interfacial barrier effects on the diffusivity/conductivity of the material. The experimental results presented in this paper only scratch the surface. There are other variations of fiber weaves and fiber orientations which have not been mentioned. Most of these variations have accompanying theoretical predictions. The shortcoming of several papers that were reviewed, in the course of research for this paper, is that they did not present respective test data to prove or disprove the theory.

There appears to be one major researcher of matrix-fiber composites at high temperature in the United States, DPH Hasselman, who works out of Virginia Polytechnic Institute and State University. Hasselman usually attempts to show a correlation between test results and the rule of mixtures theory. Hasselman has also worked to improve the laser flash test method ${ }^{3}$.

\section{Future}

The future definitely calls for testing of additional composites. A central reference location for characterizing test results as mentioned above would help designers and bring suppliers closer to market for wider use of their product. 
There currently is no method for measuring diffusivity/conductivity of single fibers or whiskers. In the absence of a method, experimenters back calculate conductivity from diffusivity values of the matrix and composite. The validity of this method could be proven by determining the diffusivity of two composites of different matrix material but identical fiber materials and then back calculating to determine the fiber conductivity. Ideally a test method to measure the diffusivity/conductivity of independent fibers or whiskers would be beneficial. The inability to instrument the sample, inability to focus the laser to a small enough diameter, and heat losses are the current testing limitations. 
References

1. WJ Parker, RJ Jenkins, CP Butler and GL Abbott, "Flash method of Determining Thermal Diffusivity, Heat Capacity, and Thermal Conductivity", Journal of Applied Physics, 1679-1684, September 1961.

2. RC Heckman, "Finite Pulse-Time and Heat-Loss Effects in Pulse Thermal Diffusivity Measurements", Journal of Applied Physics, 1455-1460, April 1973.

3. DPH Hasselman and KY Donaldson, "Effects of Detector Nonlinearity and Specimen Size on the Apparent Thermal Diffusivity of NIST 8425 Graphite", International Journal of Thermophysics, 573-585, Volume II, No. 31990.

4. JJ Brennan, LD Bensten and DPH Hasselman, "Determination of the Thermal Conductivity and Diffusivity of Thin Fibres by the Composite Method", Journal of Materials Science, 2337-2342, 17 (1982).

5. LS Han and AA Cosner, "Effective Thermal Conductivity of Fibrous Composites", Journal of Heat Transfer, 387-392, May 1981.

6. PE Phelan and RC Niemann, "Effective Thermal Conductivity of Thin, Randomly Oriented Composite Materials", Journal of Heat Transfer, 971-976, November 1998.

7. PH McCluskey, RK Williams, RS Graves, and TN Tiegs, "Thermal Diffusivity/Conductivity of Alumina-Silcon Carbide Composites", Journal of the American Ceramic Society, 461-464, 73 (2) 1990.

8. H Bhatt, KY Donaldson, and DPH Hasselman, "Role of the Interfacial Thermal Barrier in the Effective Thermal Diffusivity/Conductivity of SiC-FiberReinforced Reaction Bonded Silicon Nitride", Journal of the American Ceramic Society, 312-316, 73 (2) 1990.

9. KY Donaldson, BD Trandell, Y Lu, and DPH Hasselman, "Effect of Delamination on the Transverse Thermal Conductivity of a SiC-Fiber-Reinforced SiC-Matrix Composite", Journal of the American Ceramic Society, 1583-1588, 81 (6) 1998.

10. DPH Hasselman and LF Johnson, "Effective Thermal Conductivity of Composites with Interfacial Thermal Barrier Resistance", Journal of Composite Materials, 508-515, June 1987.

11. H Bhatt, KY Donaldson, and DPH Hasselman, " Role of Interfacial Carbon Layer in the Thermal Diffusivity/Conductivity of Silicon Carbide Fiber-Reinforced Reaction-Bonded Silicon Nitride Matrix Composites", Journal of the American Ceramic Society, 334-340, 75 (2) 1992. 
12. H Bhatt, KY Donaldson, DPH Hasselman, K Chyung, and MP Taylor, "Role of Specimen Geometry in the Effect of Fiber Orientation on the Thermal Conductivity of a Uniaxial Carbon-Fiber-Reinforced Aluminoborosilicate GlassMatrix Composite", Journal of the American Ceramic Society, 1463-1565, 74 (6) 1991.

13. G. Ziegler and DPH Hasselman, "Effect of Phase Composition and Microstructure on the Thermal Diffusivity of Silicon Nitride", Journal of Materials Science, 495-503, 161984.

14. G Zeigler, LD Bentsen, and DPH Hasselman, "Orientation Effects on the Thermal Diffusivity of Hot-Pressed Silicon Nitride", Journal of the American Ceramic Society, C35-C36, February 1981.

15. K Niihara, LD Bentsen, DPH Hasselman, and KS Mazdiyasni, "Anisotropy Effects in the Thermal Diffusivity of $\mathrm{Si}_{3} \mathrm{~N}_{4}$-BN Composites", Journal of the American Ceramic Society, C117-C118, September 1981.

16. H Tawil, LD Bentsen, S Baskaran, and DPH Hasselman, "Thermal Diffusivity of Chemically Vapour Deposited Silicon Carbide Reinforced with Silicon Carbide or Carbon Fibres", Journal of Materials Science, 3201-3212, 201985.

17. LM Russell, KY Donaldson, DPH Hasselman, ND Corbin, JJ Petrovic, and JF Rhodes, "Effect of Vapor-Liquid-Solid and Vapor-Solid Silicon Carbide Whiskers on the Effective Thermal Diffusivity/Conductivity of Silicon Nitride Matrix Composites", Journal of the American Ceramic Society, 874-877, 74 (4) 1991.

18. SR Nutt, "Defects in Silicon Carbide Whiskers", Journal of the American Ceramic Society, 428-431, 67 (6) 1984.

19. M Russell, LF Johnson, DPH Hasselman, and R Ruh, "Thermal Conductivity/Diffusivity of Silicon Carbide Whisker Reinforced Mullite", Journal of the American Ceramic Society, C226-C229, 70 (10) 1987.

20. GA Slack, "Thermal Conductivity of Pure and Impure Silicon, Silicon Carbide, and Diamond", Journal of Applied Physics, 3460-3466, December 1964. 